\title{
The Role of Stem Cells in the Treatment of Type 1 Diabetes Mellitus and Associated Complications
}

\author{
Lena Deb ${ }^{1}$, Mia Jenkins ${ }^{1}$, Malcolm Meredith ${ }^{1}$, Katelyn Rennyson \\ ${ }^{1}$ Georgetown University School of Medicine \\ Keywords: Type 1 Diabetes, Stem Cell Therapy, Insulin, Autoimmune, T1D \\ https://doi.org/10.52504/001c.29777
}

\section{Georgetown Medical Review}

Vol. 5, Issue 1, 2021

\begin{abstract}
Type 1 diabetes (T1D) affects many individuals worldwide and is associated with multiple long-term complications. The underlying pathophysiology of T1D involves an autoimmune process that leads to destruction of pancreatic $\beta$-islet cells, which are the sole cells responsible for producing the body's insulin. Thus, the current recommended treatment for T1D is insulin therapy, which requires continuous awareness and monitoring throughout the day, placing a great deal of stress and responsibility on patients. Various modalities are under investigation to provide alternatives to treatment, or even a cure. Adult endogenous progenitor cells have been studied as one potential therapy for patients with T1D due to their ability to prevent an allogenic immune response in addition to an autoimmune response. Additionally, pancreatic ductal cells and bone marrow stem cells served as one of the first areas of research that demonstrated selfprogenitor cells could regenerate pancreatic islet cells. A major limitation to stem cell therapy success is the risk of graft rejection. However, altering immune cell composition and creating physical barriers to protect the implanted cells from attack has been one successful solution to this by circumventing the pernicious immune response that is characteristic of T1D. Other limitations of these therapies include teratoma risk and the inability to reproduce the required signaling environment for cell differentiation ex vivo. Furthermore, stem cell therapy has shown promise in its applications for treatment of diabetes-associated complications including diabetic retinopathy, diabetic nephropathy, and diabetic neuropathy. This article critically reviews previous research and current progress toward stem cell treatment options for patients with T1D.
\end{abstract}

\section{INTRODUCTION}

Diabetes is one of the most prevalent health conditions in the United States. Although type 1 diabetes (T1D) and type 2 diabetes (T2D) have similar manifestations due to the long-term effects of high blood glucose, their underlying pathophysiologies are different. T1D is a condition caused by immune destruction of $\beta$-islet cells of the pancreas. These cells are responsible for producing insulin, a peptide hormone that prompts cell uptake of glucose from the blood in the liver and skeletal muscles for further carbohydrate storage and metabolism. Without sufficient insulin, as in the case of T1D, glucose builds up in the blood because it is unable to be absorbed by cells to an appropriate extent. On the other hand, in T2D, individuals are typically able to make insulin, but their tissues and cells are considered resistant; meaning that even though insulin is present, their cells do not absorb blood glucose in accordance with the amount of insulin produced and thus glucose accumulates in the blood. T2D can be accompanied by autoimmune destruction of pancreatic cells further into the disease course. In both diseases, the cells are starved of glucose and the bloodstream has an overabundance of glucose. Thus, 
the complications associated with T1D and T2D are similar in that they are caused by the chronic effect of high blood glucose and its associated damage on both blood vessels and nerves.

There is a stark difference in the prevalence between these 2 conditions: T1D has a prevalence of only $0.5 \%$ in the US compared with a prevalence of $8.5 \%$ for T2D. ${ }^{1}$ Although T1D is overall less prevalent in the US, the disease affects a higher proportion of children than does $\mathrm{T} 2 \mathrm{D}$, which is a result of the different etiologies of the 2 diseases. In fact, $50 \%$ to $60 \%$ of reported patients with T1D are younger than 16- to 18 -years old. ${ }^{2}$ The highest incidence of T1D in younger populations is among non-Hispanic White children, with a peak incidence of 32.9 for every 100000 people within the age group of 10 to 14 years. ${ }^{1}$

The most likely risk factor for T1D is genetic causes. The best evidence to support this is a study that determined that $65 \%$ of monozygotic twins who initially did not have matching T1D diagnoses eventually were found to both have diabetes. ${ }^{3}$ Another possible risk factor for T1D is high birth weight $(>4000 \mathrm{~g})$, with each 1000-g increase in weight being associated with an additional 7\% increase in risk for developing T1D. ${ }^{4}$ This increased T1D risk associated with being overweight could lead to childhood development of diabetes, because studies have shown that childhood obesity is also linked to higher rates of T1D. ${ }^{4}$ In the frame of our current understanding, when considering all risk factors, genetics and family history are the most important predictors of future development of T1D in an individual. ${ }^{5}$

\section{BACKGROUND}

T1D is a chronic disease characterized by autoimmune destruction of pancreatic islet $\beta$-cells; the sole cells responsible for the body's endogenous production of insulin. The principal role of insulin is to facilitate the sequestration of glucose from the bloodstream into cells to allow for intracellular metabolism and subsequent energy utilization. Because patients with T1D are insulin-deficient as a result of the $\beta$-cell destruction, they are consequently unable to sufficiently regulate blood glucose levels, which results in high blood glucose (hyperglycemia). Individuals with T1D can have multiple complications in both acute and chronic disease settings. Acutely, diabetic ketoacidosis is a life-threatening consequence that can occur in patients who are not properly treated with insulin or have yet to be diagnosed. ${ }^{6}$ Long-term complications of diabetes are related to the effects of chronic hyperglycemia and are typically categorized into micro- and macrovascular conditions. ${ }^{6}$ Microvascular complications consist of diabetic foot ulcers, nephropathy, retinopathy, and neuropathy, whereas macrovascular sequelae predominantly present as coronary artery disease, cerebrovascular disease, or peripheral artery disease. ${ }^{6}$ 
For individuals with T1D, daily insulin administration is essential to mitigate disease complications and avoid potentially fatal outcomes, but it is by no means a cure. 6,7 The Diabetes Control and Complications Trial (DCCT) and its longitudinal counterpart study, the Epidemiology of Diabetes Interventions and Complications (EDIC) Trial, mark 2 landmark investigations on insulin treatment for T1D. ${ }^{8}$ Collectively, these studies found that intensive insulin therapy, consisting of multiple bouts of insulin administration per day to achieve tight glycemic control, led to more favorable long-term disease outcomes with overall reduced complication rates. ${ }^{8}$ In this study, tight glycemic control was defined by normal nondiabetic glucose ranges where the goal was premeal glucose readings of 70 to $120 \mathrm{mg} / \mathrm{dL}$, 2-hour postmeal glucose readings less than $180 \mathrm{mg} / \mathrm{dL}$, a weekly 3:00 am blood glucose level greater than $65 \mathrm{mg} / \mathrm{dL}$, and a hemoglobin $\mathrm{A}_{1 \mathrm{c}}\left(\mathrm{HbA}_{1 \mathrm{c}}\right)$ level goal less than $6.5 \%$. In reality the median $\mathrm{HbA}_{1 \mathrm{c}}$ level achieved was $7 \%$; however, this still demonstrated strong efficacy in reducing diabetic complications. ${ }^{8}$ Additional studies similar to these offer hope and promise for disease control with the sole use of insulin therapy. However, achieving these ideal blood glucose levels in every patient, each day, is still very challenging and primarily lies in the hands of the patient alongside proper physician guidance. Additionally, the DCCT and EDIC trials specifically discuss adverse outcomes of intensive therapy, such as hypoglycemia and weight gain, each with their own set of consequences for the patient. Furthermore, the type, dosage, and timing of insulin administration varies per patient, by day, and even per meal, depending on the estimated quantity of glucose that will be consumed. Over time, patients must learn to adjust these parameters based on their individual physiological needs as well as their estimated intake of glucose, fat, and protein. ${ }^{7}$ Even with a lifetime of experience in self-management of insulin care, issues may arise due to an imprecise method of therapy, which requires maintenance of blood glucose levels within specific boundaries for optimal outcomes. Additionally, the daily care requirements, vigilance, and later complications of this disease are demanding, both physically and psychologically, which places a significant burden on the patient. ${ }^{6}$ Myriad factors indicate the need for further investigation to identify improved therapeutic alternatives.

Since the discovery of insulin, some advances-such as the advent of insulin pumps, ports, and inhalers-have been made in the realm of insulin administration techniques, but few alternative options for therapy have been identified. ${ }^{6,7}$ Some current alternatives to insulin therapy include islet cell transplantation, as well as adjunct therapy with oral glucose-lowering agents. ${ }^{7}$ However, these options are not currently as widely used due to limited evidence on efficacy and safety. Another unique, emerging alternative, which will be discussed throughout this review, is the use of stem cell therapy (SCT) to replace lost or damaged pancreatic islet $\beta$-cells in an effort to regenerate the body's endogenous insulin production. 
In general, stem cells are characterized by their ability to differentiate into multiple different cell types and are influenced to do so by a variety of signaling molecules and neighboring cells. ${ }^{9}$ The definition of a stem cell also encompasses both the ability of indefinite self-renewal of its respectively programmed cell type, as well as the ability "to give rise to a specialized cell type that becomes part of the healthy animal." The latter part of this definition distinguishes the characteristics of stem cells from that of cancer cells. ${ }^{9}$ More specifically, embryonic stem cells (ESCs) are pluripotent, meaning they are capable of differentiation into any type of cell in the body. ESCs are usually derived from the inner cell mass portion of the developing embryo in the early days of growth, prior to uterine implantation. ${ }^{9}$ Another newer, innovative type of pluripotent stem cells are termed induced pluripotent stem cells, which are reprogrammed, previously fully differentiated adult cells. ${ }^{9}$ On the other hand, there are multipotent stem cells, which are cells that are differentiated one step further than pluripotent stem cells. This means that multipotent stem cells can only differentiate into cells of a specific tissue in the body or into cells that arise from a specified embryonic germ cell layer (ie, endoderm, mesoderm, or ectoderm). ${ }^{9}$ Some examples of multipotent stem cells include hematopoietic stem cells (HSCs) and mesenchymal stem cells (MSCs), both of which are often extracted from bone marrow. ${ }^{9}$ Due to their broad capabilities and regenerative nature, stem cells have been used for a wide variety of therapeutic applications within the last few decades. This review will discuss the application of stem cells for treatment of T1D, as well as the treatment's limitations and further applications.

\section{Proposed Methods for Limiting Rejection of Allogeneic Stem Cell Transplants}

A major barrier to the clinical use of implanted stem cells is the risk of immune rejection and tissue damage that can follow. Because T1D is caused by autoimmune destruction of pancreatic islet $\beta$-cells, it is essential that a method is used in combination with graft replacement to prevent destruction of newly implanted pancreatic stem cells. Therefore, for this type of therapy to become a viable clinical option, the stem cell research must involve consideration of the immunologic consequences. Furthermore, the use of SCT typically requires patients to take immunosuppressive drugs, which places patients at risk for infection and other potential complications such as cancer. Therefore, a method that could circumvent the body's immune response when implanting these stem cells would be of great value because it would both protect the graft from the autoimmune response, as well as potentially reduce the need for immunosuppressive therapy.

Some studies have taken an approach to address different factors of the immune mechanism involved in graft rejection. Studies on nonobese diabetic (NOD) mice have shown that NK cells play a major role in the rejection of both allogenic passenger hematopoietic cells and allogenic HSCs in islet transplants. ${ }^{10}$ Likewise, depletion of NOD NK cells has been shown to 
prevent rejection. ${ }^{10}$ Additionally, Rapamycin is a pharmaceutical agent that can be used to reduce NK alloimmunity, but it is not as effective as NK cell depletion for avoiding graft rejection. ${ }^{10}$

T cells are also active in both alloimmune and autoimmune rejection of grafted islet cells. One study showed that the use of multipotent adult progenitor cells (MAPCs), which were harvested from donor bone marrow, caused suppression of T-cell proliferation by decreasing circulation of Th1 and Th17 cytokines. ${ }^{11}$ MAPCs are also beneficial in that they induce IL-10 secretion, which is a regulatory cytokine that decreases the immune response. ${ }^{11}$ Together, these mechanisms suppress the autoreactive effector $T$ cells, which reduces their destruction of islet cells while also promoting the proliferation of regulatory $\mathrm{T}$ cells to dampen the immune response. ${ }^{11}$

Another study that aimed to address the immune-mediated rejection of grafts took the approach of using MHC-mismatched chimerism to reverse the autoimmune reaction that is caused by memory $\mathrm{T}$ cells. ${ }^{12}$ This research came after the same laboratory demonstrated that using bone marrow transplants could reverse autoimmunity in prediabetic mice and prevent T1D from developing. ${ }^{12}$ The authors established evidence that T-cell autoreactivity and alloreactivity plays an important role in rejection of allografts. ${ }^{12}$ They also found that the MHC-mismatched chimerism was superior than MHCmatched chimerism due to the fact that it "can tolerize the radiation-resistant residual host-type $T$ cells in the periphery of autoimmune NOD recipients conditioned with myeloablative [total body irradiation and] can tolerize the autoreactive $T$ cells that also possess alloreactivity." 12

Another approach to circumventing the immune response for implanted or grafted cells is by forming a physical barrier around the transplanted cells. One study reduced initial graft loss by coating porcine islet cells with composite endothelial colony-forming cells (ECFCs) before infusion into the portal vein of nude mice. ${ }^{13}$ This was shown to significantly reduce the instant bloodmediated inflammatory reaction by decreasing the deposition of platelets, C5b-9, and CD11b+ leukocytes around the islet grafts in vivo, overall inhibiting the coagulation cascade and, therefore, reducing early graft loss. However, these porcine ECFCs only last around 4 days, thus they are not sustainable for long-term graft success. ${ }^{13}$ In this study, $30 \%$ of mice did not achieve improved nonfasting glucose levels; however, looking forward, this technology could potentially work in conjunction with dextrose sulfate or lowdose heparin to further reduce the early inflammatory response. ${ }^{13}$

Another attempt with physical barrier technology involved enclosing the generated islet cells with a biocompatible macrocapsule composed of a polyurethane-polyvinyl pyrrolidone (PU-PVP) semi-interpenetrating network. This material was designed to allow low molecular-weight substances, such as insulin and glucose, to pass through while blocking immunocytes from 
accessing the grafted cells. The barrier also functioned to inhibit the growth of fibrous tissue, which had been a negative outcome of other studies with capsules lacking PVP. ${ }^{14}$ This study specifically used human amnion-derived mesenchymal-like stem cells to create pancreatic cells, which were then transplanted into mice and resulted in normoglycemia, retention of the grafts, and no teratoma formation. ${ }^{14}$

A third technological development that has been tested involves encapsulating the pluripotent stem cells in alginate microspheres, which are derived from algae plant proteins. Once inside the alginate, the stem cells differentiate into pancreatic progenitor cells. ${ }^{15}$ While this encapsulation method does not remove the threat of immune attack, it does provide some protection to the cells once implanted into the host, which reduces the number of steps usually required for SCT and protects the cells during the differentiation phase within bioreactors. ${ }^{15}$ However, many factors are still needed to improve the viability of this technology, such as removing unfavorable surface molecules including antigens and damage-associated molecular patterns (DAMPs), before advancing to human trials. ${ }^{16}$

\section{Current Clinical Research on SCT for T1D}

Although there are no approved stem cell therapies for T1D, there is extensive research being conducted to create a stem cell approach for treating this metabolic disorder. There are numerous, active clinical trials that are examining the efficacy of stem cells in T1D, and each trial's effort allows the scientific community to learn more about T1D and SCT. In 2017, a clinical trial in California at Caladrius Biosciences, clinical trial identifier NCT02691247, was granted phase 2 status for its randomized, doubleblinded, placebo-controlled, proof-of-concept clinical trial, titled the Sanford Project T-Rex Study. ${ }^{17,18}$ This trial is investigating the use of a patient's own $\mathrm{T}_{\text {Reg }}$ immune cells by first removing the cells from the patient, augmenting the cells so that they will help regulate and prevent the autoimmune attack on pancreatic $\beta$-islet cells, and administering them back to the patient. ${ }^{18}$ This study is currently in progress and has more than 100 participants, having recently completed phase $2 .{ }^{18}$

While the Sanford trial focuses on the $\mathrm{T}_{\mathrm{Reg}}$ cells as SCT, there are other investigative trials focused on the islet cells of the pancreas. The PEC-Direct Trial, clinical trial identifier NCT03163511, a phase $1 / 2$ trial in California, used human ESCs and induced them to differentiate into pancreatic tissue that can be grafted into patients with T1D. ${ }^{19}$ The stem cells were transplanted under the skin of the patient within a pouch to allow the cells to develop into fully matured $\beta$-islet cells that were capable of secreting insulin. The pouch functioned to protect the cells from destruction by the patient's immune system. ${ }^{19} \mathrm{~A}$ key feature of this trial was the use of the PEC-Direct tool, which allowed a patient's blood vessels to make direct contact with the implanted ESCs. ${ }^{19}$ 
Another trial conducted in India investigated the efficacy of a combination SCT containing both MSCs and HSCs. ${ }^{20}$ The MSCs were derived from autologous adipose tissue samples and subsequently differentiated into insulinsecreting cells prior to reimplantation. The researchers' method included culturing the cells in a way that stimulated pancreatic conditions, thus expressing transcription factors ipf-1, pax-6, and isl-1. ${ }^{21}$ Bone marrow-derived HSCs were also extracted and combined with the MSCs prior to infusion. The stem cells were coinfused into multiple sites including the portal, thymic, and subcutaneous circulation. ${ }^{20}$ Each of these sites were specifically chosen due to the high immune tolerance of the liver, the immune-privileged location of the skin, and the ability to induce tolerance through direct thymic injection. Each of these methods were aimed to reduce immune rejection of the grafted stem cells. Likewise, the MSCs also have an intrinsic immunosuppressive property, in part due to $\mathrm{T}_{\mathrm{Reg}}$ cell induction mechanisms. ${ }^{20}$ Multiple improvements were observed on follow-up with the 10 trial participants including C-peptide levels, $\mathrm{HbA}_{1 \mathrm{c}}$ levels, and blood glucose levels. The success of this trial was largely demonstrated by the observed increase in C-peptide levels alongside a decrease in exogenous insulin requirements. These 2 findings strongly support the potential viability of this treatment in reducing the burdensome daily requirements of exogenous insulin therapy in patients with T1D. Overall, this trial represents a promising alternative and a potential future cure for individuals living with T1D. ${ }^{20}$ This trial also revealed another hurdle in the quest to make SCT a viable option: determining the best source of stem cells and methods of injection. The methods used in the study did not require immune suppression in either the autologous or allogeneic transplants, providing support for this method. ${ }^{21}$

Using stem cells to revert the destruction of pancreatic islet cells would not be useful without controlling the underlying autoimmunity. In a study by Zhao et $\mathrm{al}^{22}$ multipotent stem cells isolated from human cord blood were treated with Stem Cell Educator therapy to reverse the autoimmunity of T1D. The Stem Cell Educator is a device that separates a patient's blood through a blood cell separator, cocultures the patient's lymphocytes, and returns the newly treated autologous lymphocytes to the patient's circulation. ${ }^{22}$ This study demonstrated an improvement in the patient's immune response by improving C-peptide levels, decreasing the median $\mathrm{HbA}_{1 \mathrm{c}}$ level, and decreasing the median dose of insulin in patients with some residual $\beta$-cell function. ${ }^{22}$ The device also treated effector $T$ cells by increasing the expression of costimulatory molecules, which increased the number of $\mathrm{T}_{\mathrm{Reg}}$ cells and Th1/Th2/Th3 cytokines. ${ }^{22}$ The investigators did not histologically identify regeneration of $\beta$-islet cells, but due to the demonstrated improvement of metabolic control, the investigators were able to assume there was improvement in the number and/or function of $\beta$-islet cells. ${ }^{22}$ Zhao et $\mathrm{al}^{22}$ described their study as safe and 
feasible and were able to see improvements lasting months with only a single dose of the treatment. Further studies have the possibility to see an even greater improvement with more doses. ${ }^{22}$

Another approach to limiting the destruction of pancreatic islet cells is seen through early, interventional autologous bone marrow stem cell transplantation in patients who were recently diagnosed as having T1D. ${ }^{23}$ Investigators in this study aimed to use the immune-modulating effects of bone marrow stem cells to limit the impact of antipancreatic islet antibodies on pancreatic function. ${ }^{23}$ The basis of the immune-modulating properties of this study focused on how bone marrow stem cells are involved in limiting glutamic acid decarboxylase T-cell proliferation, which would ultimately lead to decreased levels of antipancreatic islet antibodies. ${ }^{23}$

In this study, autologous stem cells were injected into patients recently diagnosed as having T1D. The autologous stem cells were prepared by treating the patients' bone marrow with Filgrastim (a granulocyte colony-stimulating factor). ${ }^{23}$ Five days after the Filgrastim treatment, bone marrow from the patients was extracted and processed to render a sample of bone marrow stem cells to be used as the intervention for this study. ${ }^{23}$ Each patient who received the intervention was placed under general anesthesia to inject $10 \mathrm{~mL}$ of the processed bone marrow stem cells into the 6th segment of the liver. ${ }^{23}$ Although this case series only included 3 patients, the results were promising. By limiting the destructive prognosis of T1D, patients receiving the bone marrow stem cell intervention experienced lowered antipancreatic islet antibodies, lowered $\mathrm{HbA}_{1 \mathrm{c}}$ levels, and increased C-peptide levels. ${ }^{23}$ Each of these laboratory values is indicative of a better prognosis for patients with T1D. Despite the fact that the specific immunologic cause of T1D was not addressed, there is still promising potential for this therapy to delay the harmful course of the disease. Additional studies will need to examine the efficacy of this intervention and its potential to mitigate the pathophysiology of T1D.

Despite the innovation of these trials, there are still common hurdles that impede the development of successful therapy. These limitations include poor survival of islet cells, numerous adverse effects, and many issues that are associated with the required level of immunosuppression of each patient for graft survival. ${ }^{24}$ Currently, in vitro-differentiated $\beta$-cell T1D therapy is favorable due to the ability to generate a large number of cells in addition to the possibility of using patient-derived cells; however, these $\beta$ cells often do not produce enough insulin once implanted. ${ }^{25}$ Although there have been no conclusive results from the T1D stem cell trials, there is extensive research being conducted to overcome the limitations that have been discovered so far. It is the optimism of the medical community that the use of stem cells will prove useful for T1D and for other chronic medical conditions. 


\section{Further Applications of SCT for Diabetes-Associated Complications}

Further advances in SCT have generated traction and demonstrated effectiveness in expanded applications for treatment of common diabetesassociated complications. These investigative areas are focused on conditions that frequently arise in cells that are highly susceptible to the chronic hyperglycemia exposure seen in diabetes, leading most commonly to diabetic retinopathy (DR), diabetic neuropathy (DN), and diabetic nephropathy (DNP). ${ }^{6} \mathrm{DR}$ is of particular importance because it is the leading cause of blindness in developed countries worldwide, affecting an estimated $80 \%$ of patients with T1D. ${ }^{6,26}$ The pathogenesis of DR is typically characterized by chronic, low-grade inflammation; retinal endothelial cell degeneration; and decreased retinal blood flow, which ultimately leads to ischemia. ${ }^{6,26}$ The later stages of DR progression involve a paradoxical compensatory response, characterized by neovascularization, which initially creates fragile vessels, which then eventually hemorrhage resulting in further damage and, often, blindness. ${ }^{6,26}$ Patients with DR are typically categorized into 2 groups by their apparent degree of neovascularization: nonproliferative DR (NPDR) and proliferative DR (PDR). ${ }^{26}$ Currently, the standard treatments for DR are primarily aimed at slowing disease progression and include laser photocoagulation, vitrectomy, corticosteroids, and antivascular endothelial growth factor therapy, among others. ${ }^{26,27}$ Without a current cure for DR, SCT presents a potential hope for this detrimental condition through its regenerative properties. A clinical trial investigating the safety and efficacy of autologous bone marrow MSCs demonstrated improvement in visual acuity in patients with both NPDR and PDR groups. ${ }^{26}$ More markers of improvement were seen in the earlier-staged NPDR group. ${ }^{26}$ However, while therapeutic success and safety were supported with no reported short-term reactions or severe adverse events, this study lacked a control group and was conducted on a relatively small sample size, which consisted of only 17 patients, and a total of 34 eyes. ${ }^{26}$ Additionally, patients were followed up for a maximum of 6 months to assess safety, which is likely insufficient to evaluate potential occurrence of neoplastic or immune complications. Investigations with these therapeutics have primarily demonstrated success in animal models, warranting further laboratory studies to establish safety, as well as clinical trials to determine efficacy. Clinical trials are currently underway to evaluate the use of induced pluripotent stem cells (NCT03403699) and autologous bone marrow CD34+ cells (NCT01736059). Recent advances in this field, in both laboratory animal models and clinical trials, offer promise for the use of stem cells in the treatment of DR.

Another condition for which similar studies have been conducted is DNP, which is the leading cause of chronic kidney disease and accounts for half of the cases of end-stage kidney disease worldwide. ${ }^{28}$ MSCs have demonstrated efficacy in a preventive treatment role for DNP. One study in mice with T1D 
showed that MSCs played a role in the prevention of DNP alongside pancreatic $\beta$-cell regeneration. ${ }^{29}$ Seven years later, the same primary investigator demonstrated renoprotective effects of MSCs in a comparative murine trial, which found that MSC-treated mice had reduced inflammatory factors as well as a "pro regenerative microenvironment" in the kidney that preserved kidney function, despite diabetes disease status. ${ }^{29}$ Finally, another similar study demonstrated the effectiveness of MSCs in DNP progression by preventing podocyte destruction. ${ }^{30}$ These studies indicated that MSCs may offer a therapeutic solution for DNP; however, they focused primarily on the preventive aspect of care, which would not be effective in reaching the larger population of people with diabetes who are already affected by DNP. Further investigation and clinical trials are necessary before these options would be available to help patients who have already been diagnosed with DNP in an attempt to prevent further deterioration to end-stage kidney failure.

Further, stem cells have also demonstrated utility for their regenerative properties in potentially treating $\mathrm{DN}$, as well as for supporting recovery of diabetic foot ulcers, a common secondary complication of diabetic peripheral neuropathy. ${ }^{31,32}$ DN affects an estimated $60 \%$ of all patients with diabetes, and there are currently no treatments available for this highly prevalent and debilitating complication. ${ }^{32} \mathrm{DN}$ is associated with prolonged hyperglycemic conditions leading to a complex cascade of neurological degeneration and eventual sensory loss. ${ }^{31,32}$ This sensory loss partially contributes to severe ulcer formation. Studies conducted in mice have demonstrated improvement of pathologic features and symptoms of peripheral neuropathy with MSC treatment, but more studies are needed to characterize the applications of these treatments in humans. ${ }^{31,32}$ The use of stem cells for DN treatment or to aid in resolution of diabetes-associated ulcers presents a very promising option that can significantly improve quality of life for patients with T1D who have these complications.

Finally, SCT holds great potential for addressing autonomic neuropathy associated with diabetes. Autonomic neuropathy often presents with erectile dysfunction, diarrhea, and digestive dysfunction. In a small phase 1 clinical trial, 4 men received autologous bone marrow-derived MSC transplants in their corpus cavernosum. ${ }^{33}$ As a result, they reported improved erectile function, sexual desire, intercourse satisfaction, and overall satisfaction with no significant adverse effects. 33

\section{Limitations and Considerations in Treatment With SCT}

Some of the limitations to SCT have previously been discussed such as risk of teratoma formation, graft rejection, and variable life span and insulin production in vivo. However, other limitations to SCT include the lack of vast signaling relationships between cells that typically occur in the fetus during cell differentiation. These interactions have not all been identified and are not well understood; thus, they are absent when stem cells are differentiated ex 
vivo. For example, it has been shown that the renin-angiotensin system plays a role in pancreatic cell differentiation. ${ }^{34}$ Another example of these types of signals is the interplay between digestive-endocrine and pancreatic-endocrine development via FoxO1 and other signals that are likely not present in stem cell differentiation. ${ }^{35}$ Thus, creating stem cells ex vivo occurs in the absence of the normal stimuli that occur in fetal development, creating a potential point of failure for this therapy.

\section{CONCLUSIONS}

The introduction of stem cell-engineered pancreatic cells poses a risk to the patient due to the inherent immune response seen in T1D. There has been research conducted from numerous angles to address the immune rejection of grafts and injected cells. This includes methods such as depleting murine host NK cells and using MAPCs to both promote regulatory T cells with IL-10 and to suppress autoreactive effector $\mathrm{T}_{\mathrm{H}} 1$ and $\mathrm{T}_{\mathrm{H}} 17$ cells and their respective cytokines. MHC-mismatched chimerism has also been used to induce immune tolerance amongst transplant recipient mice. Additionally, there have been attempts at avoiding this immune response by creating various physical barriers to protect the injected cells, including coating porcine islet cells with composite ECFC, alginate microspheres, and PU-PVP macrocapsules.

In addition to the research that has been done on ESCs, there are clinical trials that are taking additional steps to determine the efficacy of SCT in patients with T1D. These trials, although small, show significant promise by actively assessing the placement of stem cells in patients with T1D. Most of these clinical trials deal with the common hurdles of implanted islet survival and immunosuppression to limit the body's response to the implanted islet cells.

The application of SCT to highly prevalent diabetes-associated complications has also been an area of intense investigation with somewhat more promising results than the direct application of SCT for replacement of pancreatic islet $\beta$-cells. Treatments have been trialed successfully in both animal models as well as in clinical trials for DR. ${ }^{26,27}$ Safety was upheld in a clinical trial using SCT for DR in patients as well, but had considerable limitations including a short follow-up period and a small sample size. ${ }^{26}$ Additionally, animal models have demonstrated promise in stem cell treatments for DN and for aiding the recovery from diabetic foot ulcers in patients, a common secondary complication of diabetic peripheral neuropathy. ${ }^{31,32}$ Multiple studies have also investigated the application of SCT for the treatment of DNP through various approaches. Laboratory investigations have supported the potential use of SCT for preventive care, but there is a lack of evidence for the use of SCT as a method of treatment in patients who have previously been diagnosed as having DNP. ${ }^{28,29}$ Promising evidence exists for SCT for each of these conditions, but further study and clinical trials are needed before translation into daily clinical practice can occur. If clinical trials demonstrate efficacy and safety, 
these discoveries may offer life-altering options for many individuals with T1D who have these complications and thus significantly improve the quality of life for these patients.

While this review focused mainly on the clinical trials related to SCT in regenerating pancreatic islet-cell function to produce more insulin, further research is needed in understanding the role that stem cells play in the natural pathophysiology of T1D. Although the mechanism is yet to be understood, Fadini et $\mathrm{al}^{36}$ demonstrated that episodes of mild hypoglycemia in patients with T1D affects the levels of endothelial progenitor cells (EPCs) and severe hypoglycemic episodes are associated with a marked reduction of circulating stem cells and EPCs. ${ }^{36}$ Thus demonstrating that SCT may address harmful physiologic processes at play because fluctuations in stem cells and EPCs may reduce the body's inherent healing process. Furthermore, it has been shown that MSCs are immunomodulators and migrate to the site of various injuries. ${ }^{36}$ Future research is needed to understand the role of circulating stem cells in the pathophysiology of hypoglycemia in T1D.

Considerations when engaging in SCT include the risk of teratoma formation, immune rejection, and consequent inflammation and tissue destruction, as well as potential ethical issues. Furthermore, limitations to stem cell research include the lack of signaling interaction from other cell types and organ systems during differentiation ex vivo, as well as limited life span once implanted. Overall, the breadth of application potential for stem cells in the treatment of T1D and its resulting complications offer a significant amount of promise for improved outcomes for this lifelong condition. 


\section{REFERENCES}

1. Xu G, Liu B, Sun Y, et al. Prevalence of diagnosed type 1 and type 2 diabetes among US adults in 2016 and 2017: population based study. BMJ. 2018;362:k1497. doi:10.1136/bmj.k1497

2. Daneman D. Type 1 diabetes. Lancet. 2006;367:847-858. doi:10.1016/s0140-6736(06)68341-4

3. Redondo MJ, Jeffrey J, Fain PT, Eisenbarth GS, Orban T. Concordance for islet autoimmunity among monozygotic twins. N EnglJ Med. 2008;359(26):2849-2850. doi:10.1056/nejmc0805398

4. Harder T, Roepke K, Diller N, Stechling Y, Dudenhausen JW, Plagemann A. Birth weight, early weight gain, and subsequent risk of type 1 diabetes: systematic review and meta-analysis. AmJ Epidemiol. 2009;169(12):1428-1436. doi:10.1093/aje/kwp065

5. Bingley PJ, Mahon JL, Gale EA. European Nicotinamide Diabetes Intervention Trial G: Insulin resistance and progression to type 1 diabetes in the European Nicotinamide Diabetes Intervention Trial (ENDIT). Diabetes Care. 2008;31(1):146-150. doi:10.2337/dc07-0103

6. Katsarou A, Gudbjörnsdottir S, Rawshani A, et al. Type 1 diabetes mellitus. Nat Rev Dis Primers. 2017;3(1):17017-1701717016. doi:10.1038/nrdp.2017.16

7. American Diabetes Association. 9. Pharmacologic approaches to glycemic treatment. In: Standards of Medical Care in Diabetes-2020. American Diabetes Association; 2020.

8. Nathan DM; DCCT/EDIC Research Group. The diabetes control and complications trial/ epidemiology of diabetes interventions and complications study at 30 years: overview. Dia Care. 2014;37(1):9-16. doi:10.2337/dc13-2112

9. Biehl JK, Russell B. Introduction to stem cell therapy. J Cardiovasc Nurs. 2009;24(2):98-105. doi:10.1097/jen.0b013e318197a6a5

10. Al-Adra DP, Chan WFN, Anderson CC. Nonobese diabetic natural killer cells: a barrier to allogeneic chimerism that can be reduced by rapamycin. Transplantation. 2011;92(9):977-984. doi:10.1097/tp.0b013e3182313e70

11. Reading JL, Yang JHM, Sabbah S, et al. Clinical-grade multipotent adult progenitor cells durably control pathogenic $T$ cell responses in human models of transplantation and autoimmunity.J Immunol. 2013;190(9):4542-4552. doi:10.4049/jimmunol.1202710

12. Wang M, Racine J, Zhang M, et al. MHC-mismatched chimerism is required for induction of transplantation tolerance in autoimmune nonobese diabetic recipients.J Immunol.

2014;193(4):2005-2015. doi:10.4049/jimmunol.1401137

13. Jung HS, Kim MJ, Hong SH, et al. The potential of endothelial colony-forming cells to improve early graft loss after intraportal islet transplantation. Cell Transplant. 2014;23(3):273-283. doi: $10.3727 / 096368912 \times 661364$

14. Kadam SS, Sudhakar M, Nair PD, Bhonde RR. Reversal of experimental diabetes in mice by transplantation of neo-islets generated from human amnion-derived mesenchymal stromal cells using immuno-isolatory macrocapsules. Cytotherapy. 2010;12(8):982-991. doi:10.3109/ $\underline{14653249.2010 .509546}$

15. Carroll L, Mridha AR, Tuch BE. Encapsulation and transplantation of pancreatic progenitor cells. Methods Mol Biol. 2019;2029:93-102. doi:10.1007/978-1-4939-9631-5_8

16. Krishnan R, Ko D, Foster CE, et al. Immunological challenges facing translation of alginate encapsulated porcine islet xenotransplantation to human clinical trials. Methods Mol Biol.

2017;1479:305-333. doi:10.1007/978-1-4939-6364-5_24

17. Sietsema W. Phase 2 safety and efficacy study of clbs03 autologous t-regulatory cells in adolescents with recent onset type 1 diabetes mellitus. California Institute for Regenerative Medicine. Published online 2020. 
18. CIRM. Published online 2017.

19. Clinical Trials.gov. Published online 2020.

20. Dave SD, Vanikar AV, Trivedi HL, Thakkar UG, Gopal SC, Chandra T. Novel therapy for insulin-dependent diabetes mellitus: infusion of in vitro-generated insulin-secreting cells. Clin Exp Med.2015;15(1):41-45. doi:10.1007/s10238-013-0266-1

21. Thakkar U, Trivedi H, Vanikar A, Dave S. Insulin-secreting adipose-derived mesenchymal stromal cells with bone marrow-derived hematopoietic stem cells from autologous and allogenic sources for type 1 diabetes mellitus. Cytotherapy. 2015;17(7):940-947. doi:10.1016/ j.jcyt.2015.03.608

22. Zhao Y, Jiang Z, Zhao T, et al. Reversal of type 1 diabetes via islet beta cell regeneration following immune modulation by cord blood-derived multipotent stem cells. BMC Med. 2012;10(1). doi:10.1186/1741-7015-10-3

23. Mesples A, Majeed N, Zhang Y, Hu X. Early immunotherapy using autologous adult stem cells reversed the effect of anti-pancreatic islets in recently diagnosed type 1 diabetes mellitus: preliminary results. Med Sci Monit. 2013;19:852-857. doi:10.12659/msm.889525

24. Welsch CA, Rust WL, Csete M. Concise review: lessons learned from islet transplant clinical trials in developing stem cell therapies for type 1 diabetes. Stem Cells Transl Med. 2019;8(3):209-214. doi:10.1002/sctm.18-0156

25. Nichols J, Cooke A. Overcoming self-destruction in the pancreas. Curr Opin Biotechnol. 2009;20(5):511-515. doi:10.1016/j.copbio.2009.09.009

26. Gu X, Yu X, Zhao C, et al. Efficacy and safety of autologous bone marrow mesenchymal stem cell transplantation in patients with diabetic retinopathy. Cell Physiol Biochem. 2018;49(1):40-52. doi:10.1159/000492838

27. Rong L, Gu X, Xie J, et al. Bone marrow CD133+ stem cells ameliorate visual dysfunction in streptozotocin-induced diabetic mice with early diabetic retinopathy. Cell Transplant. 2018;27(6):916-936. doi:10.1177/0963689718759463

28. Ezquer F, Giraud-Billoud M, Carpio D, Cabezas F, Conget P, Ezquer M. Proregenerative microenvironment triggered by donor mesenchymal stem cells preserves renal function and structure in mice with severe diabetes mellitus. Biomed Res Int. Published online 2015:164703. doi: $10.1155 / 2015 / 164703$

29. Ezquer FE, Ezquer ME, Parrau DB, Carpio D, Yañez AJ, Conget PA. Systemic administration of multipotent mesenchymal stromal cells reverts hyperglycemia and prevents nephropathy in type 1 diabetic mice. Biol Blood Marrow Transplant. 2008;14(6):631-640. doi:10.1016/ j.bbmt.2008.01.006

30. Wang S, Li Y, Zhao J, Zhang J, Huang Y. Mesenchymal stem cells ameliorate podocyte injury and proteinuria in a type 1 diabetic nephropathy rat model. Biol Blood Marrow Transplant. 2013;19(4):538-546. doi:10.1016/j.bbmt.2013.01.001

31. Evangelista AF, Vannier-Santos MA, de Assis Silva GS, et al. Bone marrow-derived mesenchymal stem/stromal cells reverse the sensorial diabetic neuropathy via modulation of spinal neuroinflammatory cascades.J Neuroinflammation. 2018;15(1). doi:10.1186/s12974-018-1224-3

32. Han JW, Choi D, Lee MY, Huh YH, Yoon Y. Bone marrow-derived mesenchymal stem cells improve diabetic neuropathy by direct modulation of both angiogenesis and myelination in peripheral nerves. Cell Transplant. 2016;25(2):313-326. doi:10.3727/096368915×688209 
33. Al Demour S, Jafar H, Adwan S, et al. Safety and potential therapeutic effect of two intracavernous autologous bone marrow derived mesenchymal stem cells injections in diabetic patients with erectile dysfunction: an open label phase I clinical trial. Urol Int.

2018;101(3):358-365. doi:10.1159/000492120

34. Wang L, Leung PS. The role of renin-angiotensin system in cellular differentiation: implications in pancreatic islet cell development and islet transplantation. Mol Cell Endocrinol.

2013;381(1-2):261-271. doi:10.1016/j.mce.2013.08.008

35. Carolan PJ, Melton DA. New findings in pancreatic and intestinal endocrine development to advance regenerative medicine. Curr Opin Endocrinol Diabetes Obes. 2013;20(1):1-7. doi:10.1097/ med.0b013e32835bc380

36. Fadini GP, Boscari F, Cappellari R, et al. Effects of hypoglycemia on circulating stem and progenitor cells in diabetic patients. J Clin Endocrinol Metab. 2018;103(3):1048-1055.

doi:10.1210/jc.2017-02187 